Questions de communication

$24 \mid 2013$

Renouvellement des mises en scène télévisuelles de la politique

\title{
Ce que les cultural studies font aux savoirs disciplinaires
}

Paradigmes disciplinaires, savoirs situés et prolifération des studies

What cultural studies do to Disciplinary Knowledge. Disciplinary Paradigms, Located Knowledge and Proliferation of the Studies

Éric Maigret

\section{OpenEdition}

Journals

Édition électronique

URL : http://journals.openedition.org/questionsdecommunication/8684

DOI : $10.4000 /$ questionsdecommunication.8684

ISSN : 2259-8901

Éditeur

Presses universitaires de Lorraine

Édition imprimée

Date de publication : 31 décembre 2013

Pagination : 145-167

ISBN : 978-2-8143-0182-5

ISSN : 1633-5961

Référence électronique

Éric Maigret, "Ce que les cultural studies font aux savoirs disciplinaires », Questions de communication

[En ligne], 24 | 2013, mis en ligne le 01 février 2016, consulté le 30 avril 2019. URL : http://

journals.openedition.org/questionsdecommunication/8684; DOI : 10.4000/

questionsdecommunication.8684 
ÉRIC MAIGRET

Communication, information, médias

Université Sorbonne nouvelle - Paris 3

F-75005

eric.maigret@univ-paris3.fr

\title{
CE QUE LES CULTURAL STUDIES FONT AUX SAVOIRS DISCIPLINAIRES. PARADIGMES DISCIPLINAIRES, SAVOIRS SITUÉS ET PROLIFÉRATION DES STUDIES
}

\begin{abstract}
Résumé. - Les cultural studies sont le révélateur de phénomènes culturels nouveaux, difficilement analysables par les sciences humaines qui ont été institutionnalisées il y a un siècle. Elles s'ordonnent en « paradigmes », revendiquent l'appellation d'« anti-discipline » lorsqu'elles se tournent vers les feminist et les queer studies, mais se présentent plus fréquemment comme une « interdiscipline » ou une «transdiscipline ». En se formant dans les zones entre les disciplines, qui forment un véritable no man's land depuis une cinquantaine d'années, les cultural studies participent d'un affaiblissement des barrières qui existent entre les diverses sciences de l'homme, donnant un véritable contenu à la fameuse injonction d'interdisciplinarité régulièrement vantée, mais rarement observée en pratique.
\end{abstract}

Mots clés. - Cultural studies, médias, féminisme, discipline, interdiscipline, paradigmes. 


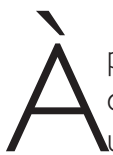
partir d'un foyer largement anglo-saxon depuis le milieu du $x x^{e}$ siècle mais qui a largement débordé depuis, la multiplication des studies dans le paysage universitaire international peut encore surprendre si ce n'est irriter. C'est en particulier le cas dans les espaces disciplinaires constitués antérieurement comme la sociologie, la science politique ou la psychologie et dans les dispositifs institutionnels nationaux qui ont parfois pu se cristalliser dans une relation privilégiée avec ces disciplines sur le mode de la résistance à l'égard de discours perçus comme menaçants (notamment en France). On connaît la liste des reproches adressés à des studies contagieuses : exagérément liées à un contexte anglo-saxon pour être transposables ailleurs, pas assez précises épistémologiquement et méthodologiquement, ne faisant que réinventer la roue quand elles parviennent à des résultats, trop engagées dans les controverses publiques voire assimilables à un simple militantisme qui détourne de la recherche, à la fois vagues car postmodernes et trop précises car hyperspécialisées... Si l'heure n'est plus aujourd'hui à la défense presque apologétique de recherches qui ont largement prouvé leur productivité face à des discours parfois un peu affolés (Maigret, 2005), il est intéressant d'utiliser ces reproches comme autant d'incitations à interroger le sens des recompositions à l'œuvre dans l'ensemble des sciences humaines, en posant des questions fondamentales comme celle de leur épistémologie, de leur statut social et politique, en bénéficiant du chemin parcouru depuis une soixantaine d'années par les cultural studies. En quoi ces dernières témoignent-elles de l'évolution des disciplines et des conceptions de la recherche dans un univers académique qui n'a pas vu se mettre en place un jeu à somme nulle (où disparaitraient les prétendues « vieilles » disciplines pour que d'autres prennent leur place) ? Comment se situentelles dans le paysage très large des studies qu'elles ne recouvrent pas? Les cultural studies s'ordonnent-elles en « paradigmes » et suivent-elles un chemin balisé en se constituant finalement en discipline, après avoir souvent revendiqué l'appellation d'« anti-discipline » ? Mais que signifient aujourd'hui cette dernière expression ainsi que celles d'« interdiscipline » et de « transdiscipline » couramment utilisées à leur propos? Quels sont les gains et les coûts de ne plus revendiquer le principe de « neutralité axiologique » ? Enfin, peut-on saisir la pertinence de la référence à des contextes nationaux et à des situations culturelles nouvelles en analysant au passage ce que révèlent les discours qui la promeuvent ou qui la taisent? Ce n'est pas le moindre des apports d'un débat approfondi sur les cultural studies que de relancer des interrogations sur l'ensemble du processus scientifique qui ne se réduisent pas à l'empilement des résultats des multiples terrains tout en se nourrissant d'eux.

\section{Les constructions nationales de la science : empirisme « britannique » et rationalisme « continental »}

Si l'on prend au sérieux l'idée de traditions nationales de recherche, il est possible de considérer l'émergence des cultural studies à Birmingham comme l'efflorescence nouvelle d'un courant philosophique profondément ancré dans une culture britannique. Avec leurs irréductibles spécificités, Raymond Williams (1957, 1961), 
Edward P. Thompson (1963) et Richard Hoggart (1957) traitent tous de pratiques culturelles traversées par des rapports de pouvoir et choisissent de privilégier une vision expressiviste, anthropologique, renvoyant à la finesse des situations étudiées, sans nécessaire effet de domination vécue, sans intégration dans un modèle « sociologique » global, plutôt qu'une vision polémologique, faite de purs rapports de force. Les références de Raymond Williams (1983) et Edward P. Thompson à David Hume et l'attirance de Richard Hoggart (1957) pour la diversité du sensible traduisent leur préférence pour un empirisme prudent, parfois romantique, au détriment d'une conception rationaliste de la recherche qui suppose l'existence d'un critère simple et universel pour évaluer l'apport des divers modèles employés. On sait que, chez David Hume, la réfutation de la métaphysique ne signifie pas un rejet des sciences, mais un rejet de leur prétention à s'unifier, contrairement au rationalisme. Pour l'essentiel, les connaissances sont un fait de croyance, car c'est dans l'esprit et non dans le réel que la cause et l'effet sont unis, par habitude. La raison n'étant qu'une variété d'instinct, elle ne peut rendre compte de sa propre démarche. C'est le non-rationnel, la répétition par habitude, qui fonde la rationalité et, par conséquent, la science elle-même, de même qu'il produit le moi comme une fiction de l'imagination, par la succession des perceptions multiples. Dans cette conception pragmatique et non spéculative de la science, la vérité est ce qui rend l'action possible et non l'adéquation à un objet.

Cette invitation à l'humilité et à la prudence, qui fait croire à la science à l'exclusion de tout principe fondateur dans la science, demeure très distinctement au cœur du projet des cultural studies et, plus largement, du processus d'élaboration des diverses studies qui ne revendiquent pas nécessairement leur appartenance à la mouvance issue de Raymond Williams, Edward P. Thompson, Richard Hoggart puis Stuart Hall. Le savoir scientifique ne peut que se pluraliser et se disséminer en une multitude d'enquêtes partielles, il y a renoncement à l'unité, mais pas à l'extension des connaissances. Cette perspective est assez courante chez des chercheurs en cultural studies qui, pour un regard extérieur formé aux divers structuralismes « continentaux », semblent parfois s'éparpiller et s'épuiser dans une infinité de sous-champs d'études, passer d'un modèle théorique à un autre (althussérien, gramscien, barthésien, lacanien, derridien, foucaldien, butlerien...) sans systématiser leurs sources, de façon qui peut paraître utilitaire. De même, l'évocation sans cesse réaffirmée de nouveaux « tournants » qui déplaceraient les enjeux par le truchement de terrains mobiles heurte de plein fouet une vision rationaliste attachée à l'idée de fondation et de système unifié par un progrès rectiligne, mais aussi une vision dite « littéraire », qui partage avec les premières cultural studies un penchant pour l'analyse du sens (le problème étant que l'analyse littéraire n'accepte pas pour autant leur défense d'une inscription dans un savoir scientifique vérifiable). Un tel positionnement épistémologique fait sécession avec le positivisme - ce frère ennemi empiriste - qui provient, lui aussi, de la philosophie humienne. Dans cette dernière, le rejet d'un système a priori unifié des savoirs n'interdit pas l'élaboration a posteriori d'un système complet des sciences incluant les sciences de l'homme, empruntant totalement 
leur méthode et leur modèle d'intelligibilité à la physique newtonienne (puis aux autres sciences de la nature). Très présent dans les sciences sociales anglosaxonnes (par exemple, en économie, en sociologie du choix rationnel ou en psychologie cognitive), le positivisme croise la philosophie analytique lorsqu'il se veut effort de rigueur linguistique, identifiant par facilité les cultural studies à un relativisme qu'elles ne défendent ni ne propagent.

De ce point de vue, la diffusion des cultural studies aux États-Unis, quoique très originales dans leurs formes, ne brise pas véritablement la trajectoire empiriste britannique, si peu préoccupée par la géométrie classique et les rigidités supposées du réel (selon la belle description de John Law, 2004), quand elle rencontre le pragmatisme philosophique d'un William James ou d'un John Dewey, qui y est solidement implanté.Attentifs à l'expérience et à ses possibilités, à la compréhension mouvante des pratiques plus qu'aux explications dernières, les travaux de James Carey (1977, 1989), pionnier des cultural studies nord-américaines, ou de Lawrence Grossberg (1988, 1992, 1995, 1997, 201 I), l'un de ses anciens étudiants passé par Birmingham devenu aujourd'hui la figure de prou du mouvement, contribuent à étendre l'influence d'une certaine façon de faire de la science : par déplacements permanents. Lawrence Grossberg lui a donné un nom, le « conjoncturalisme », et un contenu théorique sur lequel je reviendrai, en s'inspirant de Louis Althusser (1970) et de Stuart Hall (1985). II a aussi précisé son positionnement politique en défendant l'absence d'engagement dans le clivage politique droite/gauche des chercheurs des cultural studies. Les cultural studies déploient des politiques qui sont trop complexes pour intégrer une activité monodisciplinaire et pour se laisser enfermer dans une polarisation partisane classique, malgré la supériorité numérique très évidente des sympathies « liberales » ou de gauche.

Dès ses débuts, cette pratique scientifique est critiquée par des auteurs marxistes déplorant l'absence de méthodologies structurales (positivistes) et, plus encore, le manque d'engagement politique de ceux qui sont pourtant en contigüité avec leur combat. La charge de Perry Anderson $(1964,1968)$ contre la culture contestataire « médiocre » en Grande-Bretagne, par comparaison celles de la France et de l'Italie, va jusqu'à atteindre Raymond Williams et Richard Hoggart, accusés d'évacuer le conflit de leur pensée. Dans cette exploration des formes culturelles contemporaines, notamment de celles des ouvriers, que sont les premières cultural studies, se manifesterait une affinité avec la structuration politique non révolutionnaire de la Grande-Bretagne, en contraste avec une France révolutionnaire' où la sphère intellectuelle a longtemps valorisé (et continue toujours de valoriser) les ruptures

\footnotetext{
' Le phénomène de valorisation des ruptures franches par la sphère intellectuelle fait-il verser l'Allemagne du côté français ou britannique ? Même si elle n'est pas socialement révolutionnaire du point de vue historique (selon la fameuse démonstration de Theda Skocpol, 1979), la culture intellectuelle allemande est fortement contestataire et bien sûr a engendré le marxisme, en plus des autres théories totalisantes et/ou surplombantes, de Friedrich Hegel à Jürgen Habermas. Mais, l'Allemagne possède également d'importants penseurs de la conjoncture (comme Georg Simmel, repris par l'école de Chicago) et si ses institutions universitaires valorisent des disciplines fortement établies, l'ouverture à la diversité scientifique et aux phénomènes multiculturels y est plus élevée
} 
franches - et ceux qui les théorisent. La figure du penseur universaliste, voire absolutiste, et la division rigoureuse de savoirs progressivement intégrés dans une totalité, par hégélianisme devenu marxisme dans les années 50, puis par marxisme évoluant en linguistique structurale dans les années 60-70, règne d'un côté de la Manche là où l'empirisme et le fabianisme politique persistent de l'autre, aboutissant à une figure plus modeste de l'intellectuel (figure dite « organique » chez Stuart Hall, reprenant Antonio Gramsci).

On peut penser que cette division trop simple des modes de production scientifique est aujourd'hui entièrement dépassée, mais elle se manifeste toujours, au moins dans les pratiques quotidiennes de recherche, par une tendance à l'émission d'une constellation de studies d'un côté - quand le positivisme anglo-saxon accorde suffisamment d'espace à cette constellation - et par une défense de « disciplines » stabilisées de l'autre, spécifiquement en France, où la définition des savoirs est même garantie de façon para-étatique, via la production d'une séries d'instances. Les Conseil national des universités (CNU), Agence d'évaluation de la recherche et de l'enseignement supérieur (AERES) et autres mécanismes de labellisation, sélection, promotion et contrôle des savoirs et des personnes sont chargés de discipliner le rapport aux hors-disciplines et n'ont pas d'équivalent dans les pays anglo-saxons.

Après le triomphe des structuralismes en France, le courant lui aussi largement français de la déconstruction, qui détricote les postures totalisantes et les méthodologies positivistes, a pu faire croire à un rapprochement avec l'empirisme anglo-saxon - Gilles Deleuze (1953) se dit lecteur de David Hume - comme si le cartésianisme et la dialectique hégélo-marxiste s'effaçaient au profit d'une recherche par objets et par enjeux plus locaux. De façon très claire, il relève d'abord d'un reniement tardif du fondement, d'autant plus violent et excessif qu'il vient d'un espace intellectuel qui l'a survalorisé. Dans la vague postmoderniste, pourtant opposée à toute idée de fondation, les penseurs français se présentent encore en position de surplomb, sans la modestie qui sied à des chercheurs spécialisés, se battant pour des vérités partielles. De façon significative, la brouille entre Michel Foucault et Jacques Derrida prend ainsi la forme d'une critique foucaldienne de la déconstruction vue comme mouvement textualiste et immanentiste, détaché de l'empirie donc en partie régressif, et comme une " activité de "restauration" de la tradition universitaire et de l'autorité professorale » (Eribon, 1989 :214). Surprise (ou pas) des réceptions, le destin de la déconstruction est bien différent aux États-Unis (Cusset, 2003) où les œuvres de Jacques Derrida et Gilles Deleuze viennent nourrir des travaux centrés sur des objets très concrets, par exemple les corps transgenres, et en Grande-Bretagne où Stuart Hall (1998) utilise Jacques Derrida pour énoncer de nouvelles règles méthodologiques (et non tant philosophiques) intégrant la déconstruction dans une palette pluridisciplinaire assez large et assez souple.

qu'en France. Si bien que se dessine une opposition contestable et a priori désuète entre les « îles » britanniques et un « continent » fait de grands pays européens. 


\section{La structuration paradigmatique des cultural studies}

Comme le rappellent ces évocations de réceptions croisées, pour pertinente qu'elle soit, l'identification de trajectoires nationales de recherche se heurte évidemment à des limites. II existe une scène scientifique plus ou moins globalisée : les auteurs se lisent et les espaces nationaux communiquent, se fertilisent, y compris par le truchement de «malentendus » productifs. Le positionnement des trois initiateurs des cultural studies peut sembler idiosyncrasique mais, comme le soulignent désormais de nombreux chercheurs (Gibson, 2007 ; Hesmondhalgh, 2002, 2008), il ne relève pas de l'élaboration d'une britannicité fermée. À l'époque, il s'agit d'assumer tout à la fois une distance à l'égard des marxismes déterministes et élitistes, incarnés par l'école de Francfort, sans rejet du marxisme en tant que tel, et de tenir à distance une vision littéraire opposée à la prise en compte de logiques sociales. Pour ne prendre que lui, Richard Hoggart brise les préjugés les plus répandus sur la massification de la société lorsqu'il établit que les milieux ouvriers sont capables de construire une culture propre, faite de valeurs familiales et de jeux de hasard, de plaisirs immédiats et de discussions ironiques sur les médias. Ce faisant, l'auteur souligne les limites des postures humanistes classiques et des marxismes révolutionnaires qui condamnent la culture de masse et cherchent à éduquer le public en le considérant nécessairement comme aliéné. Si l'on parvient à prouver que les « damnés de la terre », ceux de l'époque industrielle, ne sont pas sous la coupe des médias, alors personne ne l'est. En conséquence, il faut renoncer aux visions élitistes et penser la culture comme quelque chose de très complexe puisque les hiérarchies sociales, le pouvoir, existent bien, sans absolument déterminer les pratiques.

Évidemment, la dimension transnationale ressort plus fortement dans les moments de systématisation théorique comme celui offert par Stuart Hall à partir des années 70-80, l'un des plus aboutis de la seconde moitié du Xxe siècle dans l'ensemble des sciences humaines. Stuart Hall conserve une partie de l'élan anthropologique fourni par Richard Hoggart et par Raymond Williams, mais il effectue une « importation » des structuralismes et des marxismes « continentaux », de Claude Lévi-Strauss à Louis Althusser en passant par Roland Barthes et Umberto Eco, en se rapprochant des modèles sociologiques qui établissent des liens contraignants entre les diverses sphères de l'existence. La recherche passe par l'identification de systèmes de représentations formant des idéologies, en relation avec des conditions économiques, politiques, genrées, raciales, subculturelles... Les cultures ne flottent pas librement, elles sont notamment reliées aux formes de pouvoir produites par les classes sociales, via des codages et des contre-codages sémiotiques (Hall, 1972). II s'agit, d'une part, de tenir à distance la théorie adornienne critique, historiquement intéressante parce qu'elle a placé les médias dans l'espace idéologique, mais qui demeure totalement aveugle à l'égard des publics et s'avère trop imprécise sur la définition du pouvoir et, d'autre part, de ne pas verser dans le positivisme américain, « scientiste » (Hall, 1982), qui apporte un contre-point utile sur la question des publics avec Paul Lazarsfeld, mais qui demeure bloqué au stade du pluralisme et du fonctionnalisme si ce n'est à celui 
du béhaviorisme. Stuart Hall développe un marxisme « humaniste » soucieux de libération, mais opposé à tout regard condescendant sur le peuple (comme Richard Hoggart et RaymondWilliams), sur les publics des médias et les diverses minorités. II fait des médias et des interprétations que les récepteurs en ont un espace de conflits de représentations et non de manipulation, d'imposition, d'obéissance. À la nuance près que les points de vue hégémoniques y sont prépondérants, ordonnés en idéologies, elles-mêmes proches de formes langagières (Roland Barthes et Umberto Eco), tout en étant menacés en permanence par l'instabilité qu'introduit l'antagonisme des points de vue, au centre comme à la périphérie du pouvoir. La théorie gramscienne de l'hégémonie qui aimante le plus profondément la réflexion de Stuart Hall (1977) est interprétée tout autant comme un prolongement du marxisme que comme une sortie de ce dernier puisque, avec elle, une dynamique historique non prévisible est introduite. Le pouvoir cesse d'être un fait premier à partir duquel se déduisent les multiples réalités pour devenir un ensemble d'effets engendrés par des alliances conjoncturelles, des conflits de représentations systématiquement produits, mais non unifiés en dernier ressort : il n'y a pas de fondation. Stuart Hall considère que le concept de domination, avec son aspect écrasant, est beaucoup moins pertinent que la palette plus riche et plus souple que sont hégémonie, consentement et conflit.

De façon intéressante, Stuart Hall (1980) évoque lui-même l'existence de « deux paradigmes » au sein des cultural studies. Le premier est empiriste et expressiviste, il met l'accent sur l'expérience, mais court le risque du « culturalisme ». Le second est structuraliste, donc en partie kantien, anti-empiriste et marxiste ; il permet de mettre en évidence les « relations déterminées », mais s'expose à une surestimation de l'unité des relations de structure. En reprenant et en se démarquant simultanément des traditions de recherche qu'il évoque ${ }^{2}$, en passant en revue leurs multiples avantages et défauts au profit d'une théorie de l'« articulation » de moments non déterminés (penchant cependant du côté du marxisme et du structuralisme), Stuart Hall réalise une synthèse théorique qui représente, de fait, le paradigme central des cultural studies pendant deux décennies, du début des années 70 au début des années 90, malgré l'évidente multiplicité de travaux, de tendances et de références qui ont pu déboucher sur des contestations plus ou moins affirmées au nom d'un marxisme plus pur par exemple (Sparks, 1974). II faut entendre par « paradigme » ce que Thomas Kuhn (1962) définit ainsi : des « matrices disciplinaires » associées à des croyances, valeurs et techniques partagées pendant un certain temps par les membres d'une communauté scientifique. II ne s'agit pas de cadres objectifs et définitifs qui constitueraient une science non interprétative, ni même de structures inconscientes singulièrement durables comme les épistémès, ce à quoi s'opposeraient tout particulièrement les membres d'une communauté cultural studies attachée à l'absence de référent ultime, à la discursivité des effets de

\footnotetext{
2 Sa recherche de l'équilibre a pu faire dire que S. Hall se présentait en funambule de la théorie culturelle (Macé, Maigret, 2007).
} 
pouvoir et aux déplacements conjoncturels. Par son attachement à la critique de formes hégémoniques « sans garanties », sa centration sur des moments séparés (mais articulés) et sa valorisation de publics actifs, le gramscisme sert de plate-forme commune à des courants de recherche parfois disparates. Avec la reformulation du concept d'« espace public » dans un sens anti-habermassien, Nancy Fraser (1992, 2005) introduit le jeu hégémonie/contre-hégémonie dans une théorie de la reconnaissance des actions des subalternes, élargissant la recherche aux théories de la justice sociale. L'étude des identités de genre peut s'inscrire dans une conceptualisation prévue au départ pour l'espace politique stricto sensu avec le concept de « masculinité hégémonique » de Raewyn Connell (1987). Si Stuart Hall s'engage dans une théorisation du postcolonial, puis du multiculturalisme ainsi que dans une critique acerbe du thatchérisme (Hall, 2008) comme modèle puissamment hégémonique quoique plurivoque, la diffusion de ses écrits aux États-Unis et en Australie va de pair avec l'essor d'un progressisme plus modéré, participatif, puisant dans certaines lectures de Michel de Certeau (1980) des raisons d'émanciper le quotidien d'une structuration de pouvoir, malgré la présence continue du pouvoir, avec John Fiske (1989a, 1989b, 1989c), Henry Jenkins $(1992,2006)$ ou John Hartley (1996). Contrairement au cliché très répandu, ces auteurs ne sont pas réellement anarchistes ni « populistes », sauf à parler d'un « populisme méthodologique » ou « thérapeutique » (Maigret, 2000) visant à défendre temporairement la cause des publics, même si une interprétation de certains de leurs travaux est possible en ce sens. Le rejet brutal dont ils font l'objet est tout autant le révélateur d'une demande préexistante de recentrage sur des effets « durs » de pouvoir qu'une justification de ce recentrage surgissant du champ de l'économie politique de la communication qui entretient des relations simultanément intimes et conflictuelles avec les cultural studies (MacGuigan, 1992 ; Kellner, 1995 ; Grossberg, 1995 ; Hesmondhalgh, 2002), d'une partie de la recherche féministe (Morris, 1990) et, plus largement, des défenseurs des théories alternatives de la gouvernementalité (Bennett, 1998). Car, comme le pressent Stuart Hall (1980) lorsqu'il évoque l'influence de Michel Foucault dans le fameux article sur les deux paradigmes cultural studies, un troisième paradigme se met progressivement en place dès les années 80 , qui, pour certains chercheurs, ne fait pas que prolonger ou infléchir sa propre synthèse, mais s'y substitue partiellement.

\section{Le foucaldisme, le féminisme et le postmodernisme : l'orientation anti-disciplinaire}

Dans les cultural studies, la réception des écrits de Michel Foucault se fait par étapes et de façon non homogène en raison, notamment, du calendrier complexe de publications, en France comme ailleurs, si bien que coexistent plusieurs usages de cet auteur. En premier lieu, l'accent placé sur les discours - entendus comme systèmes de représentations et non simplement comme systèmes de signes - détache 
l'analyse des significations de la seule linguistique et la confronte à celle des pratiques, historiquement situées. Cette tension très productive entre discours et pratiques s'intègre bien à un espace de recherche qui se donne pour objectif l'examen des relations entre cultures et pouvoirs, que l'on peut souvent considérer comme articulées par des actes communicationnels (en particulier langagiers). Ainsi, après avoir exploré majoritairement le terrain des publics, les cultural studies intègrent-elles à leur panoplie les formations discursives en documentant leurs provenances et leurs transformations, à l'exemple des porn studies initiées par Linda Williams (I 989, 2004). En second lieu, l'outillage théorique du « dispositif » permet d'aborder les phénomènes de pouvoir sous un angle plus précis, celui d'une « microphysique » se donnant les moyens de corporaliser les discours. Le dispositif peut être mis au service d'une perspective gouvernementaliste qui n'impute pas les faits de pouvoir aux seules instances étatiques et aux classes sociales, mais à un ensemble de petites disciplines décentralisées, produisant un gouvernement des corps avec les « biopolitiques ». Enfin, l'absorption du « dernier Foucault », celui d'un acheminement complexe vers le sujet par l'analyse du « souci de soi », des « techniques de soi » et de l'« esthétique de l'existence », débouche sur une éthique des moments de suspension plutôt que de sortie du pouvoir (Hunter, 1992).

Par son ampleur théorique et méthodologique, il est clair que la pensée de Michel Foucault (196I, 197I, 1976, 1984a, 1984b, 1988) pourrait être érigée en paradigme. Sa diversité interne comme ses appropriations diversifiées réduisent cette possibilité, de même que les résistances à cette tendance. Bien que, sous de nombreux angles, des frottements opèrent avec la théorisation expressivostructurale qui a constitué le soubassement des premières vagues cultural studies, il n'existe pas, à vrai dire, de paradigme clairement unifié issu des recherches de Michel Foucault se substituant brutalement au double paradigme hallien. Plus précis que le gramscisme dans sa description des effets de pouvoir parce qu'il miniaturise ce dernier et le relie au corps, le gouvernementalisme s'avère aussi de portée moins large lorsqu'il s'agit de saisir des jeux de hiérarchie et des « blocs » en voie de constitution. De nombreux débats ont cours sur les désavantages d'une position foucaldienne trop discursive (qui se présente pourtant, au départ, comme connectant pratiques et discours) et oublieuse des agrégats sociaux. Ces débats attestent de l'absence d'une « matrice disciplinaire » au sens de Thomas Kuhn (1962). D'ailleurs, des tentatives de rapprochement entre les deux grands ensembles ne s'avèrent pas impossibles: Stuart Hall (1980, 1997) entretient un dialogue continu assez constructif avec son « concurrent » et les exemples existent d'hybridation, ainsi dans le domaine de l'étude de la masculinité où les avantages de cumuler ou de sélectionner les modèles ont finement été passés en revue (Pringle, 2005). En résumé, un arc pragmatiste de modèles partiellement joints se dessine et non une intégration simple, une unification.

Mais, c'est indéniablement la posture poststructuraliste de Michel Foucault qui bouleverse le plus profondément les cultural studies, les guidant hors de l'agencement paradigmatique. L'historicisation radicale et le constructivisme 
nietzschéen sans concession de l'auteur de La volonté de savoir (1976) conduisent à une critique de l'intrication des niveaux de savoir et de pouvoir qui a pour conséquence un rejet de la prétention disciplinaire scientifique. Tout régime de vérité est nécessairement système d'exclusion discursive et refoulement vital, même s'il est aussi production de savoir (Foucault, 197I). Dès lors, il est préférable d'envisager l'activité scientifique comme une pratique historique sans cesse à interroger et à déconstruire dans ses effets de pouvoir, à l'inverse de la conception wébérienne de la « neutralité axiologique » (Weber, 1919). D'autres savoirs convergent ici vers l'attracteur foucaldien pour en renforcer l'importance. Le postmoderniste philosophique de Jacques Derrida (1968), Jean-François Lyotard (1979) et Gilles Deleuze (1968 ; Deleuze, Guattari, 1980, 1992), est un aiguillon fort de la remise en cause des catégories « naturellement » établies. Les women's studies, feminist studies, gender studies et queer studies, qui ont leur trajectoire historique propre, croisent les cultural studies à partir des années 70-80, leur fournissent une série de postures nouvelles foncièrement antidisciplinaires, pour ne plus réellement se désintriquer d'elles par la suite 3 . Leur affirmation dans l'espace nord-américain et le radicalisme de leur démarche intellectuelle tiennent à un phénomène de compensation ou de déplacement désormais bien connu : dépourvue d'une gauche radicale dans l'espace politique aux États-Unis, la scène politique contestataire s'est largement structurée dans le monde académique autour de catégories identitaires, celles de « race » et de « genre » (Davies, 1995 ; Harris, 1992, Brantlinger, 1990). À rebours du pragmatisme modéré et du positivisme conservateur, mais aussi du féminisme britannique (Gibson, 2007), le féminisme américain, à l'instar du mouvement noir, est né de la confrontation directe avec un « establishment » quasiment militarisé issu de la Guerre froide. II se conçoit comme révolutionnaire avant de s'institutionnaliser en important, dans le monde académique, une vision radicale du pouvoir et une dénonciation du rôle naturalisant des discours qui cherche, par la psychanalyse, à déchiffrer les nœuds intimes de la sujétion et du désir avant de trouver, avec le mouvement queer, dans la déconstruction derridienne et dans la théorie foucaldienne de l'assujettissement, des moyens de saper des ordres culturels hétéronormés.

Avec Donna Haraway (|99|), les féministes américaines délégitiment le discours universaliste, seul habilité à s'ériger en posture savante rationnelle, non pour défendre un relativisme populaire ou un subjectivisme, mais pour instaurer une épistémologie du positionnement où toutes et tous, humains et non-humains,

\footnotetext{
${ }^{3}$ La thèse de l'intrication des feminist, queer, postcolonial et cultural studies ne fait pas consensus (Turner, $20 \mathrm{II}$ ). Pour ma part, je revendique cette thèse car elle me semble indispensable à la bonne compréhension du projet intellectuel. Elle est défendue dans la plupart des pays qui ont développé une tradition forte de recherche dans les deux premiers domaines face à un déterminisme marxiste très hostile aux cultural studies (en France : Burch, Sellier, 2009 ; Bourcier 200 I, 2005, 201 I ; Dorlin, 2006, 2008 ; Guénif-Souilamas, Macé, 2006; Cervulle, Rees-Roberts, 2010 ; Cervulle, 2013 ; Quemener, à paraitre). Mais, cette thèse peut conduire à ne pas respecter l'autonomie de développement des savoirs comme dans les derniers travaux de Marie-Hélène Bourcier (20 I I) laissant entendre qu'il est impossible de pratiquer les cultural studies si l'on est homme, blanc et hétérosexuel, dans une vision queer ghettoïsée des cultural studies qui surprendrait R. Hoggart, R. Williams - et S. Hall le premier.
} 
cessent d'être désincarnés et se voient dotés de compétences inégales en fonction de leur accès aux mondes. II s'agit de valoriser les « savoirs situés », ceux des non-savants, ou ce que Donna Haraway nomme encore la figure du « témoin modeste » face au « privilège de la perspective partielle », lorsqu'une cécité trop grande affecte les savoirs savants comme dans le cas de la structuration patriarcale de la science ${ }^{4}$. S'il n'existe pas de neutralité scientifique, l'objectivité demeure atteignable par la mise en relation des partialités, socialement constituées et non seulement subjectivement vécues, au cours d'une collaboration produisant le savoir rationnel - la démarche rappelle celle de Bruno Latour $(1999,2006)$ par influences réciproques. Dans ses ouvrages sur les effets performatifs des discours genrés, Judith Butler (1990, 1997a, 1997b) ne nie en rien l'existence du sexe biologique, mais l'historicise tant sur le plan social que du point de vue d'une nature qui n'est plus essentialisée. La déconstruction ne sert pas à démontrer que tout est discursivement construit, mais que les équivalences entre sexes et genres produisent de l'exclusion et du stigmate, lesquels perturbent, à leur tour, les ordres discursifs en exhibant leur arbitraire. $\grave{A}$ une profusion de discours normatifs doit répondre une «prolifération » toute foucaldienne d'identités et de contre-discours, y compris dans l'espace universitaire où, par leur attention aux situations vécues, les studies deviennent autre chose que de l'empirisme sauvagement pluriel : un antidote aux formes de pouvoir ${ }^{5}$. La perspective déborde le pragmatisme et conduit les cultural studies sur la voie d'un constructivisme que l'on pourrait qualifier de « critique » et de « participatif » et non plus de « social » (au sens de Peter Berger et Thomas Luckmann, 1966). Judith Butler (1990) souligne distinctement le fait qu'un décloisonnement opère entre espaces scientifiques et mouvements sociaux, entre recherche et pratique contestataire, ou du moins culturelle, relativisant les efforts d'intégration paradigmatique de Stuart Hall, même si le concept d'« hégémonie » demeure revendiqué (Butler, Laclau, Žižek, 2000) :

« La même question se pose inlassablement : celle de savoir si mon travail, ou celui de Homi Bhabha, Gayatri Chakravorty Spivak ou de Slavoj Žižek, relève des cultural studies ou de la théorie critique. Mais le fait de poser pareille question pourrait simplement indiquer que la distinction claire et nette entre ces deux types de projets ne tient plus. On trouvera des théoricien-ne-s pour prétendre que l'ensemble de nos travaux relève des cultural studies, mais on trouvera aussi des spécialistes dans ce domaine pour se définir contre toute forme de théorie (même si de manière significative, ce n'était pas le cas de Stuart Hall, l'un des fondateurs des cultural studies en Angleterre). Mais quel que soit le parti pris dans ce débat, les un-e-s et les autres ne réalisent pas toujours que la théorie a changé de visage en faisant précisément l'objet d'appropriations culturelles. La théorie s'est trouvé un nouvel espace, nécessairement impur, où elle émerge dans et par la traduction culturelle et comme le produit de celle-ci » (Butler, 1990 : 28-29).

\footnotetext{
${ }^{4}$ Les effets de normalisation produits par la science sont particulièrement visibles sur le terrain transgenre, analysé par Éric Macé (2010).

${ }^{5}$ La politique foucaldienne de prolifération d'identités et de contre-discours est également travaillée parTeresa de Lauretis (1987) et Eve Kosofsky Sedgwick (1991).
} 


\section{Le sens et la portée de l'interdisciplinarité et de la postdisciplinarité}

Le spectacle donné par les cultural studies dans les années 2000 peut s'avérer surprenant par la coexistence de deux tendances a priori contradictoires. La consolidation disciplinaire se poursuit avec la production de manuels et de synthèses sur l'histoire de la recherche (par exemple : Couldry, 2000 ; Hartley, 2000 ; During, 2005 ; McRobbie, 2005 ; Rojek, 2007 ; Lewis, 2008 ; Walton, 2012). Comme le souhaite Tony Bennett (1998) qui plaide pour une codification disciplinaire, une perspective résolument additive sinon intégratrice conduit à canoniser les théories et à associer les références venues de disciplines ou de courants parfois très éloignés: histoire, philosophie, psychanalyse, psychologie, sciences politiques, sémiotique, sociologie... En Australie, une véritable institutionnalisation accompagne ce mouvement sur fond de politique multiculturelle défendue par l'État et de défense du rôle citoyen des industries culturelles, assimilant les cultural studies à des cultural policy studies de plus en plus critiquées pour leur normalisation (During, 2005). Mais, ailleurs, l'implantation est beaucoup plus complexe et des effets de retour en arrière ne sont pas absents, avec la disparition symbolique du centre de Birmingham (Webster, 2004). Sur le plan théorique, le trouble introduit par le postmodernisme, le poststructuralisme et le féminisme tient à distance le spectre positiviste, accentue la fragmentation et le pluralisme des cultural studies, déjà très présents à leurs débuts. La contradiction entre normalisation et déconstruction pourrait mener à un constat d'échec si elle ne se dissipait pas dans une redéfinition des cultural studies non comme discipline installée, mais comme activité d'identification des relations fluctuantes entre pouvoirs et cultures et comme exercice de connexion entre disciplines à mobiliser pour surmonter les nouvelles formes d'assujettissement. Cette orientation transparaît dès le milieu des années 80 des écrits de Stuart Hall $(1986,2007)$, unissant le marxisme althussérien à ce que l'on doit, nommer faute de mieux, une « anglicité » parfois non contrariée. II faut rappeler tout à la fois l'urgence de l'action face à un désir de théoriser pouvant tourner à vide, l'autonomie des « conjonctures » sociales, toujours laborieuses à appréhender, et ne pas généraliser à outrance : penseur des « relations déterminées 》 et importateur du structuralisme, Stuart Hall est en réalité beaucoup plus empiriste qu'il ne le pense ou ne l'avoue. De nouvelles questions émergent sans cesse de « nouvelles conjonctures historiques », nécessitant de nouvelles réponses que les disciplines établies ne peuvent automatiquement apporter. II faut reconnaître à Stuart Hall (1989, 1992) une mise en adéquation avec cette visée qui aborde, tour à tour, les luttes liées à la race et à l'ethnicité, le multiculturalisme et le postcolonial, dans des contextes britanniques précis, bien avant que les problèmes de racisme et de cohabitation culturelle ne soient placés au sommet de l'agenda de la plupart des sciences sociales établies. Ce que Lawrence Grossberg (1997, 20 I I) appelle dans la continuité de Stuart Hall une « discipline de contextualité », un « contextualisme radical » ou un « conjoncturalisme » devient l'objet et l'objectif perpétuel des cultural studies, leur visée de savoir-pouvoir et leur style 
propre. II soude une communauté scientifique qui ne s'accorde véritablement que sur la possibilité de diverger dans un espace commun aux frontières épistémologiquement floues car productivement contestables, effectuant comme une mise en abîme de la théorie de la sphère publique au sein même de l'espace scientifique qui l'a engendrée. Pour reprendre len Ang (2007), les cultural studies interviennent là où sont requis de façon intrinsèque des niveaux d'analyse multidimensionnels, où le contingent et le complexe l'emportent. Elles se présentent comme une pluridiscipline ou, plus précisément, comme une « (inter)discipline postmoderne » au sens de Vincent B. Leitch (2003). II faut entendre par là une activité de savoir cumulatif́ filtrant des marges et des supposées incompatibilités entre disciplines généralement établies au début du $X X^{e}$ siècle, hybridant les outils de ces dernières lorsqu'ils s'avèrent suffisamment conciliables et féconds. En opérant ainsi, les cultural studies jouent le rôle d'un programme intégrateur sans aller jusqu'à instituer une discipline nouvelle prenant la place de celles qui les auraient précédées (Johnson et al., 2004).

La redéfinition postdisciplinaire des cultural studies n'intervient pas hors de toute conjoncture universitaire et sociale. Elle répond à un contexte nouveau dont elle est le symptôme et l'un des meilleurs analystes. En effet, on pourrait relire l'histoire des sciences sociales depuis un siècle à l'aune d'une ossification continue des savoirs, après l'effervescence des premiers moments d'exploration et de codification. Également observée dans les sciences de la nature, cette trajectoire banale, « normale » car efficiente, conduit à la séparation progressive de savoirs spécialisés puis isolés. Or, ce processus devient insoutenable à partir du moment où les spécialités sont frappées d'incommunication, où l'ossification devient fossilisation, dans une situation de multiplication de nouveaux phénomènes difficilement appréhendables comme ceux que connaissent les sociétés de la modernité avancée ou seconde (flux transnationaux disjonctifs, identités plus instables voire détachées des ancrages socio-économiques ${ }^{7}$, variable des classes sociales insuffisante pour expliquer les actions et représentations, loisirs généralisés, etc. $)^{8}$. Les cultural studies naissent de la chute de rendement scientifique des disciplines. Elles sont en résonance avec le «tournant culturel » qu'elles ont incontestablement identifié, justifiant une appréhension nouvelle de l'empirie contemporaine et, logiquement, un réaménagement trans ou postdisciplinaire.

\footnotetext{
${ }^{6}$ II faut mesurer ici la proximité et la distance avec Jean-Claude Passeron (|99|), dont on connait la description non popperienne des sciences sociales. La postdiscipline cultural studies procède par déplacements, mais elle produit également des fusions conceptuelles et des intégrations paradigmatiques tout en décloisonnant les espaces universitaires et non universitaires.

7 Les affinités entre mouvements culturels et facteurs socio-économiques ne sont plus envisageables comme des corrélations statistiques univoques, mais comme des séries de problèmes en partie autonomes, comme dans le cas du hip hop et du capitalisme contemporain ou dans celui de la culture metal (Guibert, 2000 ; Guibert, Sklower, 20 I3).

${ }^{8}$ Les limites voire l'épuisement des sciences sociales contemporaines sont bien dessinées par John Urry (2000) ou Michel Wieviorka (2007). Le concept de modernité dite « seconde » ou « réflexive » est issu des travaux d'Anthony Giddens (1990) et Ulrich Beck (1986), celui de « flux disjonctifs » des travaux d'Arjun Appadurai (1996).
} 
Ce changement avait été anticipé par Edgar Morin qui, dans La méthode (1977), rappelait le contrepoint indispensable d'un travail de spécialisation, la nécessité heuristique mais aussi démocratique de raccorder les savoirs, non pour réinstaller le philosophe dans la position de «Prince de la Science », opérant la grande synthèse, mais pour mettre fin à la violence des discours séparés. « II s'agit d'encyclo-péder, c'est-à-dire d'apprendre à articuler les points de vue disjoints du savoir en un cycle actif » (Morin, 1977 : 19), l'effort portant non sur la totalité des connaissances de chaque sphère, mais sur les « points stratégiques, les nœuds de communication, les articulations, organisationnelles entre les sphères disjointes 》. Formulé autrement, avec Joe Moran (200I), une réorganisation des savoirs est requise lorsque les disciplines deviennent excluantes, lorsqu'elles ne sont plus que des chasses gardées défendues par des institutions érigées en systèmes de pouvoir. En termes de sujets abordés comme de méthodes apportées par les réflexions postdisciplinaires, à partir d'interrogations universitaires ou extrauniversitaires (les « savoirs situés 》), la créativité encourage la communication et de nouvelles alliances entre disciplines ainsi que la critique réflexive. De plus, elle ouvre aux disciplines existantes de nouvelles perspectives, ce qui explique l'absence de substitution entre cultural studies et disciplines, cadres usuels d'organisation des savoirs?.

Non sans ironie, la remarque sur l'essoufflement des disciplines peut se révéler pertinente pour la constellation cultural studies elle-même, qui n'échappe pas à l'hyperspécialisation de studies de moins en moins liées entre elles (quelles passerelles entre game studies, nostalgia studies et food studies ?) et à des durcissements conceptuels locaux (dont ont été coutumières les women's et les feminist studies). Bien peu suffit pour que les chercheurs oublient le défi de l'élaboration de conceptions théoriques intégrant les divers espaces disciplinaires, revenant à une routine bien connue. Les difficultés de la profusion modélisatrice s'ajoutent à celles de la spécialisation outrancière. L'ouverture transdisciplinaire peut pousser à faire circuler des schémas non maîtrisés, à manier des concepts qui n'ont pas la même portée et signification d'une discipline à l'autre. Pour autant, les risques de confusion généralisée, d'ingestion par une métadiscipline gloutonne ainsi que d'annexion d'une discipline par une autre sont aujourd'hui moins élevés que les risques de solitude perpétuelle et de rejet de l'une par l'autre. En se formant dans les zones entre les disciplines, qui forment un véritable no man's land depuis une cinquantaine d'années, puis en passant des marges au centre, les cultural studies participent d'un affaiblissement des barrières qui existent entre les diverses sciences de l'homme, donnant un véritable contenu à la fameuse injonction d'interdisciplinarité régulièrement vantée, mais rarement observée en pratique.

\footnotetext{
${ }^{9}$ L'absence de confrontation frontale entre sociologie et cultural studies, au profit d'une succession de moments disciplinaires et interdisciplinaires, a été présentée dans l'épais volume dirigé par Elisabeth Long (1997), regroupant la fine fleur de la sociologie américaine de la culture et des médias.
} 


\section{Conclusion}

Dans la contribution, j'ai souvent cherché à souligner ce que les cultural studies doivent à leur lieu de création et à celui de leur propagation première, respectivement la Grande-Bretagne et les États-Unis, non pour verser dans une sorte de culturalisme qui réduirait des savoirs à une structure éternellement contraignante, mais pour comprendre le rôle des facteurs locaux dans la montée d'un savoir à prétention objective. Dans la formulation des enjeux théoriques et méthodologiques, l'empirisme et le pragmatisme, qui ne sont pas sans rapport avec l'absence de tradition révolutionnaire en Grande-Bretagne, ont joué un rôle majeur en contribuant à la définition d'une figure non élitiste de l'intellectuel, sans rupture absolue avec un « peuple » qui serait pour sa part dépossédé de ses expériences de vie. Au contraire, les revendications quasiment révolutionnaires des féministes américaines (et des mouvements Black Panthers, ici non abordés) ont incité à critiquer de façon radicale les effets de naturalisation, sans rompre pour autant avec le primat de l'expérience. Enfin, ce n'est pas un hasard si l'appel d'air postdisciplinaire que sont les cultural studies provient, au départ, des espaces académiques anglo-saxons où les complexes obsidionaux sont moins marqués qu'en Europe « continentale ». Le précédent de la psychologie sociale, qui est née par pragmatisme du constat de l'impossibilité de séparer de façon pure des faits psychologiques réservés au psychologue et des faits sociaux réservés au sociologue, est parlant. Cette grille de lecture par tradition nationale pourrait voir son usage prolongé avec l'évocation toujours pertinente des relations complexes entre l'Australie et la Grande-Bretagne, entre la Grande-Bretagne et ses anciennes colonies, sans négliger les complémentarités et rivalités implicites entre espaces anglo-saxons et français ou encore l'absence de réflexivité de la recherche américaine dans les années qui ont suivi le I I septembre 200 | puis la crise financière de 2008. Mais, pour conclure, je préfère envisager ce qui ne relève plus du local dans la recherche cultural studies, ce qui est de l'ordre de la montée en généralité, si l'on veut bien accepter qu'une discipline de la conjoncture ne se limite pas à un ensemble de déplacements immanents.

Dans cette optique, puisqu'il faut bien naître quelque part, les cultural studies sont nées en Grande-Bretagne de la geste fondatrice de Richard Hoggart dans les années 60, puis du travail d'extension des fondations de Stuart Hall. Mais les cultural studies ne sont plus - et même ne sont pas - une forme de pensée anglo-saxonne représentant une école très institutionnalisée, avec ses figures incontestées et son savoir bien délimité. Si les cultural studies doivent quelque chose à un espace-temps spécifique, comme tout courant de recherche, elles ont depuis longtemps dépassé cet instant zéro en se diversifiant géographiquement et intellectuellement, en s'appropriant également l'héritage d'un nombre important de penseurs qui ne relèvent pas de la tradition d'origine, enfin, en refusant l'idée d'un savoir figé, d'une clôture. Cela signifie-t-il alors qu'elles s'éparpillent en tous sens, en perdant toute scientificité ? La réponse est négative. La diversification ne s'est pas faite au détriment d'une véritable logique de recherche unifiée : 
les cultural studies ne sont pas dépourvues d'idées directrices, ni de méthodes rigoureuses. Leur spécificité est tout simplement de refuser de s'ériger en discipline pour mieux épouser/contrarier la dynamique fluctuante du monde. Elles sont un art de faire et de défaire les pouvoirs et les identités, se donnant pour objectif une émancipation non naïve des formes de vie. Les luttes de représentation sont incertaines dans les médias comme ailleurs et débouchent parfois sur la promotion partielle de points de vue marginaux qui demeurent traversés de points de vue hégémoniques. La pertinence paradigmatique du modèle de Stuart Hall est évidente, modèle peu à peu enrichi et dépassé, y compris par ce même auteur, du terrain des soap operas à celui de la téléréalité, en passant par l'expression sur l'internet ou la pornographie.

Intéressées par les médias parce qu'ils sont le grand terrain de jeu de la production des identités et des pouvoirs, les cultural studies ne sont pas pour autant médiacentriques. Elles s'élargissent dans les années 1980-2000 à l'ensemble des revendications culturelles. II n'existe pas de tabou sur les objets à traiter car il faut saisir l'espace public comme constitué par les conflits de représentation et non comme un espace neutre vers lequel convergeraient les questions politiques. Les quêtes de représentation et de participation des ouvriers, des femmes, des jeunes, des homosexuels, des minorités ethnoraciales, bref de ceux que l'on nomme désormais les subalternes, façonnent et refaçonnent une sphère publique très plastique qui est tout simplement l'ensemble des questions faisant sens à un moment donné dans la société (souvent via les médias de masse). En procédant ainsi, les cultural studies rejoignent ou enfantent en grande partie les gender studies, les postcolonial studies, les queer studies... Elles s'ouvrent aux reformulations des sciences humaines et sociales qui, du poststructuralisme au postmodernisme en passant par la déconstruction, affirment la possibilité d'un jeu ontologique, l'existence d'un principe d'indétermination, donc la possibilité de surmonter une condition de subalterne tout en n'étant pas inféodé en permanence aux identités. La remise en cause des frontières supposées naturelles entre les formes culturelles, entre leurs supports et entre les formes de médiation symbolique devient le plus marquant des mouvements intellectuels des deux dernières décennies. Les segmentations entre œuvres majeures et mineures sont débordées de toutes parts, comme celles entre culture supposée légitime et culture dite populaire, dans un contexte de prolifération des objets dignes d'être étudiés et enseignés. Au-delà, ce sont les frontières jusque-là glacées du masculin et du féminin qui subissent le soupçon et le réchauffement de la controverse publique comme scientifique : les genres et leur dénaturalisation sont devenus des thèmes transversaux autant que des modes de réflexion sur les disciplines elles-mêmes. De la même façon, ancrée dans l'analyse de ce qui ne passe pas dans les relations anciennement coloniales, la critique postcoloniale (Bhabha, 1994) fonctionne à la fois comme démarche herméneutique infléchissant les sciences humaines dans leur ensemble et comme espace empirique, collection de terrains en littérature, en théâtre, en histoire, en communication... 
En résumé, cet art de faire et de défaire les identités qui est développé par les cultural studies n'est pas seulement un « sport de combat » qui consisterait à lever le voile des illusions pour les mystifiés et à prendre le pouvoir à la façon des penseurs marxistes classiques (de Francfort à Paris pourrait-on dire, c'est-à-dire de Theodor Adorno à Pierre Bourdieu). II s'agit d'apprendre des subalternes, déjà porteurs de sens et de capacités d'agir, et de leur donner les moyens symboliques pour qu'ils se constituent contre ce qui les nie - moment de l'« essentialisme stratégique » théorisé par Gayatri Spivak (1988, 1999), moment du faire de la négritude, des mouvements gays et lesbiens, des publics de fans - avant qu'ils ne rejettent la violence même de leur émancipation - moment du défaire des identités, devenues trop rigides et asservissantes : il n'existe pas de monde noir unifié et essentiel, de lesbianisme ghetto, de catégorie stable de fans. Parfois, le rejet radical de la fondation qui soutient les réflexions des cultural studies est considéré comme une voie sans issue. Comment imaginer un monde commun si rien ne semble tenir? Tous les critiques ne sont pas des chercheurs nostalgiques du savoir absolu (et de la position qui va avec), cette objection est donc importante. D'ailleurs, elle émane des rangs même des cultural studies où elle joue un rôle très productif. Mais, elle passe sous silence le fait qu'une critique tout aussi importante et parfaitement opposée est aussi régulièrement émise, celle d'adhérer à une théorie du multiculturalisme qui ferait des groupes des identités fermées sur ellesmêmes, essentialisées. Relativistes ou essentialistes les cultural studies? La véritable question ne se pose pas en ces termes. La plupart des auteurs considèrent tout simplement, à la façon de Donna Haraway et de Judith Butler, qu'il n'y a d'accès à l'universel que par le particulier, et que cet universel n'est pas une totalité ultime. II faut donc distinguer des cycles : des moments où des définitions essentielles sont imposées aux individus et aux groupes, des moments où l'émancipation peut consister en un cri identitaire essentialisant et des moments de rejet des identités essentielles, tout en n'oubliant jamais les conditions socio-économiques qui aident ou qui contrecarrent ces mouvements, tout en les produisant pour partie (pour partie seulement). Impossible d'oublier que ce sont souvent des minorités aisées qui articulent la parole des subalternes, que des limites matérielles à l'émancipation existent, que les replis identitaires et les politiques racistes changent avec les conjonctures économiques. II y a donc nécessité de procéder par « anti-essentialisme », mais aussi au moyen d'un « anti-anti-essentialisme » voire d'un « anti-anti-anti-essentialisme », pour reprendre la terminologie de Paul Gilroy (1992, voir aussi 1987, 2004). Sans remettre en cause le projet intellectuel, un ralliement au matérialisme est observé et jugé bienvenu dans les cultural studies début de siècle, avivé par un rejet du postmodernisme ${ }^{10}$ et par le sentiment que les points de vue un peu éthérés, « élitistes » ou « eurocentriques » ne peuvent avoir cours par temps de guerre et de crise économique"'. Le renouveau de la critique

${ }_{10}$ On mesurera le rejet du postmodernisme au gré du chemin parcouru par Angela McRobbie (1994, 2008).

"Le sentiment que les points de vue « élitistes » ne peuvent avoir cours par temps de guerre et de crise économique n'est pas universellement partagé, y compris par les nouvelles générations qui 
qui escorte cette re-sociologisation n'est pas sans sympathie avec les mouvements anticapitalistes (Gilbert, 2008) et avec l'autonomisme italien (Lazzarato, 2000 ; Hardt, Negri, 2000, 2004) posant la question de l'oscillation permanente entre détermination et compréhension à laquelle seraient vouées les cultural studies ou celle d'une sortie progressive de cette oscillation vers un nouveau paradigme de topologie immanente du social (Baruch Spinoza, Michel Foucault et Gilles Deleuze plutôt qu'Antonio Gramsci et Richard Hoggart) susbstituant l'affect au duo sens/ plaisir (Ahmed, 2010 ; Gregg, Seigworth, 2010), non sans un retour à... David Hume. Au cours de ce nouveau «tournant » le projet cultural studies ne semble guère remis en cause, se présentant comme un prolongement et non comme une négation des Lumières, même si l'opposition trop simple entre nature et culture est repoussée vers l'horizon nouveau des minorités non humaines à libérer, s'élargissant par une succession de chocs internes et externes au profit d'un (post) humanisme universel et durable.

\section{Références}

Ahmed S., 2010, The Promise of Happiness, Durham, Duke University Press.

Althusser L., 1970, « Idéologie et appareils idéologiques d'État », pp. 269-374, in :Althusser L., Sur la reproduction, Paris, Presses universitaires de France, 1995.

Anderson P., 1964, « Origins of the Present Crisis », New Left Review, 23, pp. 27-53.

— 1968, « Components of the National Culture », New Left Review, 50, pp.3-57.

Ang I., 2007, « Cultural Studies », in: Bennett T., Frow J., dirs, The Sage Handbook of Cultural Analysis, Oxford, Sage.

Appadurai A., 1996, Après le colonialisme. Les conséquences culturelles de la globalisation, trad. de l'américain par F. Bouillot et H. Frappat, Paris, Payot, 2001.

Beck U., 1986, La société du risque. Sur la voie d'une autre modernité, trad. de l'allemand par L. Bernardi, Paris, Aubier, 200 I.

Bennett T., 1998, Culture. A Reformer's Science, Sydney, Allen and Unwin.

— 2007, « Making Culture, Changing Society », Cultural Studies, 21/4-5, pp. 61 0-629.

Berger P., Luckmann T., 1966, La construction sociale de la réalité, trad. de l'américain par P.Tamiaux, Paris, Méridiens Klincksieck, 1986.

Bhabha H. K., 1994, Les lieux de la culture. Une théorie postcoloniale, Payot, 2007.

Bourcier M.-H., 200 I, Queer Zones I. Politiques des identités sexuelles, des représentations et des savoirs, Paris, Éd. Amsterdam, 2006.

— 2005, Sexpolitiques. Queer zones 2, Paris, Éd. La Fabrique.

— 201 I, Queer Zones 3. Identités, cultures, politiques, Paris, Éd. Amsterdam.

peuvent considérer que ce back to basics cache une injonction de conformisme qui n'atteint pas son but, saisir les méandres du social et du politique contemporain (Hall, Birchall, 2006). 
Brantlinger P., 1990, Crusoe's Footprints. Cultural Studies in Britain and America, Londres, Routledge.

Burch N., Sellier G., 2009, Le cinéma au prisme des rapports de sexe, Paris, Vrin.

Butler J., 1990, Trouble dans le Genre. Pour un féminisme de la subversion, trad. de l'américain par C. Kraus, Paris, Éd. La Découverte, 2005.

— 1993, Ces corps qui comptent. De la matérialité et des limites discursives du sexe, trad. de l'américain par C. Nordmann, Paris, Éd. Amsterdam, 2009.

- 1997a, La vie psychique du pouvoir, trad. de l'américain par B. Matthieussent, Paris, L. Scheer, 2002.

- 1997b, Le Pouvoir des mots. Politique du performatif,trad. de l'américain par C. Nordmann, Paris, Éd. Amsterdam, 2004.

Butler J., Laclau E., Žižek S., 2000, Contingency, Hegemony, Universality. Contemporary Dialogues on the Left, New York, Verso.

Carey J., 1977, « Mass Communication Research and Cultural Studies : An American View », in : Curran J., Gurevitch M., Woollacott J., dirs, Mass Communication and Society, E. Arnold/The Open University Press.

- 1989, Communication as Culture. Essays on Media and Society, Londres, Routledge.

Certeau M. de, 1980, L'invention du quotidien I. Arts de faire, Paris, Gallimard, 1990.

Cervulle M., 20 I3, Dans le blanc des yeux. Diversité, racisme et médias, Paris, Éd. Amsterdam.

Cervulle M., Rees-Roberts N., 20 I0, Homo Exoticus. Race, classe et critique queer, Paris, A. Colin/Ina.

Connell R.W., 1987, Gender and power, Sydney, Allen and Unwin.

Couldry N., 2000, Inside Culture : Reimagining the Method of Cultural Studies, Londres, Sage.

Cusset F., 2003, French Theory : Foucault, Derrida, Deleuze \& Cie et les mutations de la vie intellectuelle aux États-Unis, Paris, Éd. La Découverte.

Davies I., 1995, Cultural Studies and Beyond. Fragments of Empire, Londres, Routledge.

Deleuze G., 1953, Empirisme et subjectivité. Essai sur la nature humaine selon Hume, Paris, Presses universitaires de France.

- 1968, Différence et répétition, Paris, Presses universitaires de France.

Deleuze G., Guattari F., 1980, Mille Plateaux, Paris, Éd. de Minuit.

- 1991, Qu'est-ce que la philosophie?, Paris, Éd. de Minuit.

Derrida J., 1968, « La différance », in :Tel Quel, Théorie d'ensemble, Paris, Éd. Le Seuil, repris dans Derrida J., pp. I-29, Marges de la Philosophie, Paris, Éd. de Minuit, 1972.

Dorlin E., 2006, La matrice de la race. Généalogie sexuelle et coloniale de la nation française, Paris, Éd. La Découverte.

— 2008, Sexe, genre, sexualités. Introduction à la théorie féministe, Paris, Presses universitaires de France.

During S., 2005, Cultural Studies. A Critical Introduction, Londres, Routledge.

Eribon D., 1989, Michel Foucault, édition revue et enrichie, Paris, Flammarion, 201 I.

Fiske J., 1989a, Understanding Popular Culture, Boston, Unwin Hyman. 
— 1989b, Reading the Popular, Boston, Unwin Hyman.

— 1989c, « Moments de télévision : ni le texte ni le public », trad. de l'américain par C. Jaquet, pp. 190-21 I, in : Glevarec H., Macé É., Maigret É., éds, Anthologie cultural studies, Paris, A. Colin/Ina, 2008.

Foucault M., 1961, Histoire de la folie à l'âge classique, 2e édition, Paris, Gallimard, 1972.

— 197I, L'ordre du discours, Paris, Gallimard.

— 1976, Histoire de la sexualité, t. I, La volonté de savoir, Paris, Gallimard.

— 1984a, Histoire de la sexualité, t. 3, Le souci de soi, Paris, Gallimard.

— 1984b, « Sexe, pouvoir et la politique de l'identité », pp. 1554-1565, in : Foucault M., Dits et écrits, 1976-1988, vol. II, Paris, Gallimard, 2001.

— 1988, « Les techniques de soi », pp. 1602-1632, in : Foucault M., Dits et écrits, 19761988, vol. II, Paris, Gallimard, 2001.

Fraser N., 1992, « Repenser la sphère publique : une contribution à la critique de la démocratie telle qu'elle existe réellement », trad. de l'américain par M.Valenta, Hermès, 31, 200 I, pp. 109- 142.

- 2005, Qu'est-ce que la justice sociale? Reconnaissance et redistribution, trad. de l'américain par E. Ferrarese, Paris, Éd. La Découverte.

Gibson M., 2007, Culture and Power. A History of Cultural Studies, Oxford, Berg.

Giddens A., 1990, Les conséquences de la modernité, trad. de l'anglais par O. Meyer, Paris, Éd. L'Harmattan, 1994.

Gilbert J., 2008, Anti-Capitalism and Culture. Radical Theory and Popular Politics, Oxford, Berg.

Gilroy P., 1987, There Ain't No Black in the Union Jack. The Cultural Politics of Race and Nation, Londres, Hutchinson.

- 1992, L'Atlantique Noir. Modernité et double conscience, trad. de l'américain par J.-P. Henquel, Paris, Kargo, 2003.

— 2004, After Empire. Melancholia or Convivial Culture ?, Londres, Routledge.

Gregg M., Seigworth G. J., dirs, 20 I 0, The Affect Theory Reader, Durham, Duke University Press.

Grossberg L., éd., 1986, « On postmodernism and Articulation : An Interview with Stuart Hall », pp. 131-150, in : Morley D., Chen K. H., éds, Stuart Hall : Critical Dialogues in Cultural Studies, Londres, Routledge, 1996.

— 1988, It's a Sin. Essays on Postmodernism, Politics and Culture, Sydney, Power Publications.

— 1992, We Gotta Get Out of this Place. Popular Conservatism and Postmodern Culture, New York, Routledge.

— 1995, «Cultural Studies Vs. Political Economy : Is Anybody else Bored with this Debate? », Critical Studies in Mass Communications, I, vol. 12, pp. 72-81.

— 1997, Bringing It All Back Home. Essays On Cultural Studies, Durham, Duke University Press.

— 20I I, Cultural Studies in the Future Tense, Durham, Duke University Press.

Guénif-Souilamas N., Macé É., 2006, Les féministes et le garçon arabe. Paris, Éd. de l'Aube.

Guibert G., 2000, « L'éthique hip hop et l'esprit du capitalisme », Mouvements, I I, pp. 54-59. 
Guibert G., Sklower J., 2013, « Dancing with the devil. Panorama des « metal studies ». Accès : http://www.laviedesidees.fr//MG/pdf/20 | 3 | I 05_metal.pdf. Consulté le 07// I/ / 3.

Hall S., 1972, « Codage/décodage », trad. de l'anglais par M. Albaret et M.-C. Gamberini, pp. 25-40, in : Glevarec H., Macé É., Maigret É., éds, Anthologie cultural studies, Paris, A. Colin/Ina, 2008.

— 1977, « La culture, les médias et l'"effet idéologique" », trad. de l'anglais par C. Jaquet, pp. 4I-60, in : Glevarec H., Macé É., Maigret É., éds, Anthologie cultural studies, Paris, A. Colin/lna, 2008.

— 1980, « Cultural studies : deux paradigmes », pp. 81-104, in : Hall S., 2008, Identités et cultures. Politiques des cultural studies, trad. de l'anglais par C. Jacquet, Paris, Éd. Amsterdam.

— 1982, « Le redécouverte de "l'idéologie" : retour du refoulé dans les médias studies », pp. 129-168, in : Hall S., 2008, Identités et cultures. Politiques des cultural studies, trad. de l'anglais par C. Jacquet, Paris, Éd. Amsterdam.

— 1985, « Signification, représentation, idéologie :Althusser et les débats poststructuralistes 》, trad. de l'anglais par C. Jacquet, K. Lindner et B. Quélennec, Raisons politiques, 48-4, 20 I2, pp. $13|-| 62$.

— 1989, « Nouvelles ethnicités », pp. 287-297, in : Hall S., 2008, Identités et cultures. Politiques des cultural studies, trad. de l'anglais par C. Jacquet, Paris, Éd. Amsterdam.

— 1992 «Quel est ce "noir" dans "culture populaire noire" ? », pp. 299-310, in : Hall S., 2008, Identités et cultures. Politiques des cultural studies, trad. de l'anglais par C. Jacquet, Paris, Éd. Amsterdam.

— 1997, «The Work of Representation », pp. 13-74, in : Hall S., éd., Representation : Cultural Representations and Signifying Practices, Londres, Sage.

— 1998, « Identité culturelle et diaspora 》, pp. 3 I I-325, in : Hall S., 2008, Identités et cultures. Politiques des cultural studies, trad. de l'anglais par C. Jacquet, Paris, Éd. Amsterdam, 2008.

— 2007, « Entretien avec Stuart Hall par Mark Alizart », pp. 45-91, in : Alizart M., Hall S., Macé É., Maigret É., Stuart Hall, Paris, Éd. Amsterdam.

— 2008, Le populisme autoritaire, trad. de l'anglais par C. Jacquet, H. Saulvage, É. Beerlham, Paris, Éd. Amsterdam.

Hall G., Birchall C., dirs, 2006, New Cultural Studies. Adventures in Theory, Edinburgh, Edinburgh University Press.

Haraway D., 1991, Manifeste cyborg et autres essais. Sciences, fictions, féminismes, anthologie établie par Allard L., Gardey D., Magnan N., Paris, Exils Éd., 2007.

Hardt M., Negri A., 2000, Empire, Londres, Harvard University Press.

- 2004, Multitude. Guerre et démocratie à l'âge de l'Empire, trad. de l'américain par N. Guilhot, Paris, 10-18, 2006.

Harris D., 1992, From Class Struggle to the Politics of Pleasure. The Effects of Gramscianism on Cultural Studies, Londres, Routledge.

Hartley J., 1996, Popular Reality: Journalism, Modernity and Popular Culture, New York, Bloomsbury.

— 2000, ed., American Cultural Studies. A Reader, Oxford, Oxford University Press.

Hesmondhalgh D., 2002, The Cultural Industries, Londres, Sage. 
— 2008, « Industries culturelles et cultural studies », trad. de l'anglais par C. Jaquet, pp. 275-294, in : Glevarec H., Macé É., Maigret É., éds., Anthologie cultural studies, Paris, A. Colin/lna.

Hoggart R., 1957, La Culture du pauvre. Étude sur le style de vie des classes populaires en Angleterre, trad. de l'anglais par J.-C. Garcias et J.-C. Passeron, Paris, Éd. de Minuit, 1970.

Hunter I., 1992, « Aesthetics and Cultural Studies », pp. 347-367, in : Grossberg L., Nelson C. Treichler P., éds, Cultural Studies, New York, Routledge.

Jenkins H., 1992, Textual Poachers. Television Fans and Participatory Culture, New York, Routledge.

— 2006, La culture de la convergence. Des médias au transmédia, trad. de l'américain par C. Jaquet, Paris, A. Colin/Ina, 2013.

Johnson R., Chambers D., Raghuram P., Tincknell E., 2004, The Practice of Cultural Studies, Londres, Sage.

Kellner, D., 1995, Media Culture : Cultural Studies, Identity and Politics between the Modern and the Postmodern, Londres, Routledge.

Kuhn T. S., 1962, La Structure des révolutions scientifiques, trad. de l'américain par L. Meyer, Paris, Flammarion, 1983.

Latour B., 1999, Politique de la nature. Comment faire entrer les sciences en démocratie. Paris, Éd. La Découverte.

— 2006, Changer de société. Refaire de la Sociologie, Paris, Éd. La Découverte.

Lauretis T. de, 1987, Théorie queer et cultures populaires : de Foucault à Cronenberg, Paris, Éd. La Dispute, 2007.

Law J., 2004, After Method. Mess in Social Science Research, New York, Routledge.

Lazzarato M., 2000, «Travail et capital dans la production des connaissances », pp. I53172, in : Azais C., Corsani A., Dieuaide P., dirs, Vers un capitalisme cognitif. Entre mutations du travail et territoires, Paris, Éd. L'Harmattan.

Leitch V. B., 2003, Theory Matters, New York, Routledge.

Lewis J., 2008, Cultural Studies, Londres, Sage.

Long E., dir., 1997, From Sociology to Cultural Studies. New Perspectives, Oxford, Blackwell.

Lyotard, J.-F., 1979, La condition postmoderne. Rapport sur le savoir, Paris, Éd. de Minuit.

MacGuigan J., 1992, Cultural Populism, Londres, Routledge.

Macé É., 2010, « Ce que les normes de genre font aux corps/Ce que les corps trans font aux normes de genres », Sociologie, 4, vol. I.

Macé É., Maigret É., 2007, « Stuart Hall, le Noir de la famille », in : Alizart M., Hall S., Macé É., Maigret É., Stuart Hall, Paris, Éd. Amsterdam.

Maigret É., 2000, « Les trois héritages de Michel de Certeau. Un projet éclaté d'analyse de la modernité », Les Annales HSS, 3, pp. 5 | I-549.

— 2005, «Après le choc cultural studies », pp. 17-40, in : Maigret É., Macé É., dirs, Penser les médiacultures. Nouvelles pratiques et nouvelles approches de la représentation du monde, Paris, A. Colin/Ina.

McRobbie A., 1994, Postmodernism and Popular Culture, Londres, Routledge.

— 2005, The Uses of Cultural Studies, Londres, Sage. 
— 2008, The Aftermath of Feminism. Gender, Culture and Social Change, Londres, Sage.

Moran J., 200 I, Interdisciplinarity, New York, Routledge.

Morin E., 1977, La méthode, t. I, La nature de la nature, Paris, Éd. Le Seuil.

Morris M., 1990, « Banality in Cultural Studies », pp. 14-43, in : Mellecamp P., dir., Logics of Television : Essays in Cultural Criticism, Bloomington, Indiana University Press.

Passeron J.-C., 1991, Le raisonnement sociologique, Paris, Nathan.

Pringle R. 2005, « Masculinities, Sport and Power. A Critical Comparison of Gramscian and Foucaldian Inspired Theoretical Tools », Journal of Sport and Social Issues, 3, vol. 29, pp. 256-278.

Quemener N., à paraître, Le pouvoir de l'humour. Politique des représentations dans les médias en France, Paris, A. Colin/Ina.

Rojek C., 2007, Cultural Studies, Cambridge, Polity.

Skocpol T., 1979, États et révolutions sociales. La révolution en France, en Russie et en Chine, trad. de l'américain par N. Burgi, Paris, Fayard, 1985.

Sedgwick E. K., 1991, Épistémologie du placard, trad. de l'américain par M. Cervulle, Paris, Éd. Amsterdam, 2008.

Sparks C., 1974, «The Abuses of Literacy », Working Papers in Cultural Studies, vol. 6, pp. 7-24.

Spivak, G. C., 1988, Les subalternes peuvent-elles parler?, trad. de l'anglais par J.Vidal, Éd. Amsterdam, 2009.

— 1999, A Critique of Postcolonial Reason. Toward a History of the Vanishing Present, Harvard University Press.

Thompson E. P., 1963, The Making of the English Working Class, New York, Vintage.

Turner G., 201 I, What's Become of Cultural Studies?, Londres, Sage.

Urry J., 2000, Sociologie des mobilités. Une nouvelle frontière pour la sociologie ?, trad. de l'anglais par N. Burch, Paris, A. Colin, 2005.

Walton D., 2012, Doing Cultural Theory, Londres, Sage.

Weber M., 1919, Le Savant et le politique, trad. de l'allemand par C. Colliot-Thélène, Paris, Éd. La Découverte, 2003.

Webster F., 2004, «Cultural Studies and Sociology at, and after, the Closure of the Birmingham School », Cultural Studies, 6, vol. I8, nov., pp. 847-862.

Wieviorka (en collab. avec Debarle A. de, Ohana J.), dir., 2007, Les sciences sociales en mutation, Paris, Éd. Sciences humaines.

Williams L, 1989, Hard Core. Power, Pleasure and the "Frenzy of the Visible », Berkeley, University of California Press.

— dir., 2004, Porn Studies, Londres, Duke University Press.

Williams R., 1957, Culture and Society : 1 780-1950, Londres, Chatto and Windus.

- 1961, The Long Revolution, Londres, Chatto and Windus.

_ 1983, « David Hume : Reasoning and Experience », pp. I21 - I4I, in :Williams R., Wrinting in Society, Londres, Verso. 